\title{
Frontiers of Liver Surgery
}

\author{
Yuzo Yamamoto ${ }^{a}$ Karl J. Oldhafer ${ }^{b, c}$ \\ a Department of Gastroenterological Surgery, Akita University Graduate School of Medicine, Akita, Japan; \\ ${ }^{b}$ General and Visceral Surgery, Surgical Oncology, Asklepios Hospital Barmbek, Hamburg, Germany; \\ ${ }^{\mathrm{c}}$ Semmelweis Medical Faculty, Campus Hamburg, Hamburg, Germany
}

In the past, liver surgery has had key opportunities to undergo a dramatic evolution. These were supported by several innovative discoveries and inventions. The establishment of detailed knowledge of liver anatomy played a critical role in the development of liver surgery. As epoch-making events in anatomy, the discovery of the vasculo-biliary sheath (Francis Glisson, 1654), the definition of the right and left liver by Rex-Cantlie's line (Hugo Rex and James Cantlie, 1888 and 1889), the discovery of the hepatic venous system (Ton That Tung, 1939), as well as the identification of liver segments according to the portal system (Claude Couinaud, 1954) can be named. Regarding the aspect of surgical technique, cross-clamping of the hepatic pedicle (James Hogarth Pringle, 1908), preliminary vascular control (Jean-Louis Lortat-Jacob, 1952), and the finger fracture method (Tien-Yu Lin, 1960) formed the basis for the present procedures. This precise knowledge of anatomy and the corresponding techniques created modern liver surgery, e.g. anatomical subsegmentectomy (today called segmentectomy according to the IHPBA Brisbane 2000 nomenclature) in combination with ultrasound-guided dye injection (Masatoshi Makuuchi, 1984) and Glissonean approach (Ken Takasaki, 1990). Besides these developments, strategies to protect the function of liver remnant were described. In addition, total hepatic vascular exclusion under hypothermic perfusion (Joseph G. Fortner, 1974), ex-situ liver resection (Rudolf Pichlmayr, 1989), anterior approach (Kazue Ozawa, 1990), and liver hanging maneuver for anterior approach (Jacques Belghiti, 2001) were also noteworthy technical innovations.

Furthermore, developments in order to assess the hepatic functional reserve and to augment the volume of liver remnant largely contributed to the prevention of post-hepatectomy liver failure and thus to the safety of liver surgery. However, the most recent epochmaking innovation in liver surgery seems to be the ALPPS (associating liver partition and portal vein ligation for staged hepatectomy) procedure - initially introduced as 'in-situ split' liver resection - which made a vast impact in 2011. Since this procedure enables an astonishing increase of volume in the future liver remnant, it fascinated innovative surgeons immediately. However, as time for testing and improvement is required for every new technology, the ALPPS procedure must also undergo the process of evaluation and refinements as well as the analysis of its mechanism in order to find its proper place in the armamentarium of liver surgery, especially in relation to the already established portal vein embolization (PVE).

For this special topic of Visceral Medicine, we requested inventors of the ALPPS procedure [1] and of PVE [2] to disclose the behind-the-scenes story of invention in the light of a teaching by Confucius: 'Look for the old so as to learn the new' (fig. 1). These recounts may provide us with hints for overcoming the unresolved challenges. Additionally, we asked an expert radiologist of PVE as well as two driving forces of the ALPPS procedure in the world to contribute articles on the state-of-the-art technique of PVE to obtain an optimal hypertrophy of the liver [3], on the relationship between hypertrophy and liver function in ALPPS [4], and on the pitfalls in PVE and ALPPS [5]. Moreover, since the modalities of assessing the functional reserve of the liver differ between Western and Eastern countries, we asked two experts in the field from each area to delineate the currently most reliable methods $[6,7]$. Finally, the Hamburg group summarized the impact of the first International ALPPS Expert Meeting 2015 [8].

When reading the articles of this special topic, you will easily recognize that there is still a slight difference in tone regarding the understanding of

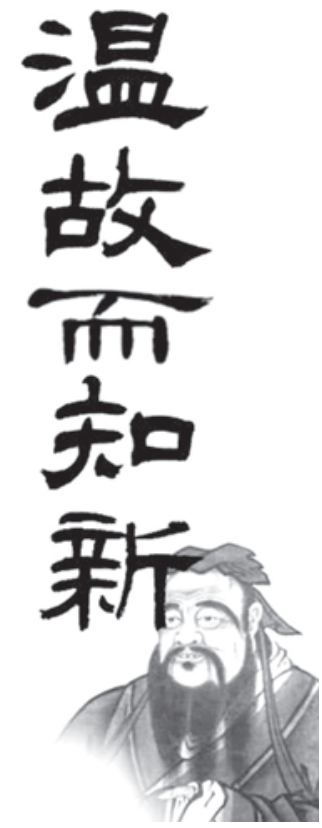

Fig. 1. 'Look for the old so as to learn the new' (teaching by Confucius).

\section{KARGER}

(๑) 2017 S. Karger GmbH, Freiburg

Fax +497614520714 
the features of the ALPPS procedure even among experts. While being someone who vehemently recommends various indications of the ALPPS procedure, Professor Schlitt, the inventor of ALPPS, humbly advises that such an invasive medical technology should be applied, with correct recognition of its absolute value and field of application, but not as a 'fashion of the times'. Thus, it can be well understood that liver surgeons carefully foster this new procedure while having a clear understanding of the risks and challenges involved. In contrast, there would be a need to internationally standardize the evaluation modalities of hepatic functional reserve in order to analyze the imbalance between the speed of volume increase and the speed of functional augmentation in ALPPS as well as in PVE, because international cooperation is absolutely neces- sary in the case of such a procedure which is not performed by many health professionals.

By means of this special topic, we are hoping that the readers correctly rate the values of ALPPS and PVE from the interdisciplinary viewpoint of expertise in surgery, internal medicine, radiology, oncology, and even pathophysiology. This will help in finding an answer regarding the proper area of application of these techniques without delay and result in a further favorable development. Consequently, liver surgery contributes to saving and extending the lives of many critically ill patients with advanced liver tumors.

Finally, the guest editors would like to express their sincere gratitude to the authors involved who provided such valuable contributions to this special topic on 'Frontiers of Liver Surgery'.

\section{References}

1 Schlitt HJ, Hackl C, Lang SA: 'In-situ split' liver resection/ALPPS - historical development and current practice. Visc Med 2017;33:DOI: 10.1159/000479850.

2 Shinkawa H, Takemura S, Tanaka S, Kubo S: Portal vein embolization: history and current indications. Visc Med 2017;33:DOI: 10.1159/000479474.

3 Huang SY, Aloia TA: Portal vein embolization: stateof-the-art technique and options to improve liver hypertrophy. Visc Med 2017;33:DOI: 10.1159/000480034.
4 Kang D, Schadde E: Hypertrophy and liver function in ALPPS: correlation with morbidity and mortality. Visc Med 2017;33:DOI: 10.1159/000479477.

5 Schnitzbauer AA: A comparison of pitfalls after ALLPS stage 1 or portal vein embolization in smallfor-size setting hepatectomies. Visc Med 2017;33:DOI: 10.1159/000480100.

6 Rassam F, Olthof PB, Bennink RJ, van Gulik TM: Current modalities for the assessment of future remnant liver function. Visc Med 2017;33:DOI: 10.1159/000480385.
7 Iimuro Y: ICG clearance test and 99mTc-GSA SPECT/CT fusion images. Visc Med 2017;33:DOI: 10.1159/000479046.

8 Stavrou GA, Donati M, Fard-Aghaie MH, Zeile M, Huber TM, Stang A, Oldhafer KJ: Did the International ALPPS Meeting 2015 have an impact on daily practice? The Hamburg Barmbek experience of 58 cases. Visc Med 2017;33:DOI: 10.1159/000479476. 